\title{
Using the yeast gene deletion collection to customize gene expression
}

\author{
Dritan Liko, Matthew G. Slattery, Chasity L. Phillips, and Warren Heideman \\ University of Wisconsin, Madison, WI, USA
}

BioTechniques 40:728-734 (June 2006)

doi 10.2144/000112192

The pioneering sequencing of the Saccharomyces cerevisiae genome (1) formed the basis for genome-wide, large-scale approaches to studying gene function. One such project was the $S$. cerevisiae Genome Deletion Project that systematically replaced each yeast open reading frame (ORF) with a kanamycin resistance module (KanMX), yielding precise start-to-stop codon deletions for more than $95 \%$ of the genes $(2,3)$. This replacement is widely used for phenotypic analysis of gene function. We have taken advantage of the thousands of KanMX-promoter fusions generated by the yeast deletion project to create insertion modules for replacing a normal promoter with any one of thousands of different possible yeast promoters.

This method takes advantage of the DNA templates produced by the gene knockout project, each possessing a specific ORF that has been replaced by a KanMX module (Figure 1). Since the flanking regions for the deleted ORFs are left intact, what remains produces a set of strains carrying unique DNA templates in which almost any intergenic region is flanked by a KanMX marker. For almost any yeast promoter, there exists a strain in which a selectable marker has been inserted immediately $5^{\prime}$ of that promoter. This KanMX marker-intergenic region can be PCRamplified as a cassette, and flanking by homologous recombination (4). used as a source of template DNA. To intended insertion sites (40-60 bp). This can be accomplished with a single PCR step. In the results shown herein, we used genomic DNA as the template, but we have also used the colony PCR method (5), which could potentially allow high-throughput workflows.

Our schematic shows the KanMXpromoter cassette inserted between the normal promoter and the ORF, but the upstream primer can be designed to cause replacement of the normal promoter sequence. We have successfully used both methods. This concept could also be used to design insertion fragments to replace $3^{\prime}$ untranslated sequences with those from another gene, as well as to make in-frame fusions between practically any two coding regions.

PCR fragments containing the KanMX-promoter cassette were amplified using genomic DNA from the chosen deletion strain (Table 1) purchased from Research Genetics (Huntsville, AL, USA).

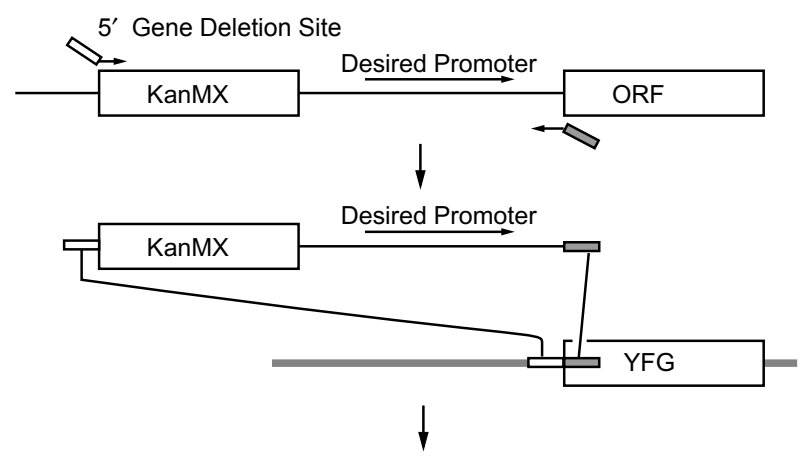

PCR using primers with 40 bp $5^{\prime}$ ends homologous to desired insertion sites sequences can be designed to target the product for insertion at the desired site

The ORF immediately $5^{\prime}$ to the promoter of interest is identified so that the knockout strain for which this ORF has been replaced by KanMX can be target the PCR product for insertion, the amplification primers are designed with $5^{\prime}$ ends that are homologous to the

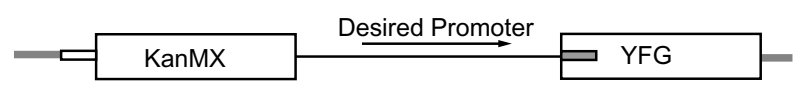

Select for recombinants with kanamycin plates

Figure 1. Amplification of a desired promoter region for insertion upstream of your favorite gene (YFG). Primers are selected to amplify the promoter of interest along with the KanMX marker that has been inserted immediately $5^{\prime}$ to the promoter in a specific strain of the gene deletion set. Amplification primers have regions at the $5^{\prime}$ ends that are homologous to the target site (shown as small rectangles). Amplified products are transformed into the strain of interest, and the PCR products are inserted by homologous recombination via the targeting sequences. $\mathrm{ORF}$, open reading frame.

Transform PCR product into desired strain for homologous recombination 
As an example of this technique, we used the CCR4 primer set and template DNA from strain 10388 (carrying a deletion in yal020c) to produce a KanMX-CCR 4 promoter fragment that was targeted to replace the normal promoter sequence upstream of the $C L N 3$ ORF. We chose the CCR4 promoter as a potential constitutive promoter because $C C R 4$ messenger RNA (mRNA) levels, encoding a general transcriptional regulator (6), were relatively constant in published microarray experiments (7). Replacement of the CLN3 promoter with that from $C C R 4$ reduced CLN3 mRNA levels and blunted CLN3 induction by nutrients (8) (Figure 2A). Thus, this method was effective in manipulating expression of the target gene.

We also used the LSRI promoter, which normally drives transcription of the U2 small nuclear RNA (snRNA) (9), as a test promoter. We chose U2 RNA as a loading control because it is constant and abundant. The KanMXLSRI promoter cassette was amplified with a DNA template from strain 13371 (carrying a deletion in $y b r 231 c$ ) using the $L S R I$ primers for insertion immediately $5^{\prime}$ of the $R P B 4$ ORF. That produced overexpression of $R P B 4$ mRNA, which encodes a subunit of RNA polymerase II (10) (Figure 2B).

To make a conditional promoter fusion, we used the $S M F 3$ promoter. $S M F 3$ encodes an iron transporter that is induced in low iron environments $(11,12)$. An $S M F 3$ promoter fusion driving CLN3 was constructed with prior amplification of the KanMXSMF3 promoter fragment using genomic DNA from strain 16433 as a template (strain 16433 carries KanMX inserted in place of $y / r 035 c)$. CLN3 mRNA levels were determined in mid-log cultures grown in either the absence or presence of an iron chelator, bathophenanthroline-disulfonic acid (BPS). As expected, BPS increased CLN3 mRNA in the altered strain (Figure 2C). The SMF3 promoter is just one example out of the hundreds of regulated promoters that could be selected for use.

Since expression can also be regulated through translational regulatory elements in the mRNA,

Table 1. Yeast Strains

\begin{tabular}{|lll|}
\hline Strain & Description & Reference \\
\hline BY4742 & MAT $\alpha$ his3 4 1 leu2 40 lys2 $\Delta 0$ ura3 $\Delta 0$ & Research Genetics \\
10388 & BY4742 carrying yal020c::KanMX & Research Genetics \\
13371 & BY4742 carrying ybr231c::KanMX & Research Genetics \\
16433 & BY4742 carrying ylr035c::KanMX & Research Genetics \\
11597 & BY4742 carrying yor301w::KanMX & Research Genetics \\
DP2 & BY4742 carrying CCR4-CLN3::KanMX & This study \\
DLY2 & BY4742 carrying LSR1-RPB4::KanMX & This study \\
DLY4 & BY4742 carrying SMF3-CLN3::KanMX & This study \\
DLY5 & BY4742 carrying CPA1-CLN3::KanMX & This study \\
\hline
\end{tabular}

we moved the $5^{\prime}$ noncoding region of CPA1 to regulate production of CLN3. Translation of Cpa1 is inhibited by arginine through regulation of ribosomal scanning of an upstream ORF $(13,14)$. The CPAl promoter driving $C L N 3$ fusion was constructed as above, amplifying the $\mathrm{KanMX-CPAI}$ promoter fragment out of strain 11597 (carrying a deletion in yor $301 w$ ). The addition of arginine $(40 \mathrm{mM})$ to the medium decreased cell size in the transformants, which was the expected phenotype for decreased Cln3 levels (not shown).

It is commonly useful to drive expression of a gene of interest with a heterologous promoter. While vectorbased chromosomal insertion tools for yeast, such as the STIK system (15), have been available for many years, our method takes advantage of the previously accomplished generation of thousands of KanMX-promoter fusions, providing ready-made templates for generating a targeted insert with a single PCR. The availability of these templates obviates the need for the intermediate step of creating a recombinant clone carrying the promoter fused to a marker. The collection of $S$. cerevisiae deletion strains provides a set of readily amplified KanMX markers lying immediately $5^{\prime}$ to intragenic regions that include approximately $95 \%$ of the promoters in S. cerevisiae. This provides a useful set of ordered templates that can be used to substitute almost any yeast promoter into a site driving almost any yeast gene. This method involves only a single PCR amplification and a standard yeast transformation. Furthermore, the growing body of microarray data allows predictions to be made regarding the strength of a given promoter under different circumstances, which should enable custom expression patterns to be readily designed.

\section{ACKNOWLEDGMENTS}

The authors wish to acknowledge Dominic Porcaro, Bruce Bagley, Michael Dapp, and Solange Eloundou for assistance, as well as B.G. Bird for helpful suggestions. This work was supported by National Science Foundation grant no. 0235379.

\section{COMPETING INTERESTS STATEMENT}

The authors declare no competing interests.

\section{REFERENCES}

1. Goffeau, A., B.G. Barrell, H. Bussey, R.W. Davis, B. Dujon, H. Feldmann, F. Galibert, J.D. Hoheisel, et al. 1996. Life with 6000 genes. Science 274:546-567.

2. Winzeler, E.A., D.D. Shoemaker, A. Astromoff, H. Liang, K. Anderson, B. Andre, R. Bangham, R. Benito, et al. 1999. Functional characterization of the $S$. cerevisiae genome by gene deletion and parallel analysis. Science 285:901-906.

3. Giaever, G., A.M. Chu, L. Ni, C. Connelly, L. Riles, S. Veronneau, S. Dow, A. LucauDanila, et al. 2002. Functional profiling of the Saccharomyces cerevisiae genome. Nature 418:387-391.

4. Rothstein, R.J. 1983. One-step gene disruption in yeast. Methods Enzymol. 101:202-211.

5. Akada, R., T. Murakane, and Y. Nishizawa. 2000. DNA extraction method for screening yeast clones by PCR. BioTechniques 28 : 668-674.

6. Denis, C.L. and J. Chen. 2003. The CCR4NOT complex plays diverse roles in mRNA 
Table 2. PCR Primers

\begin{tabular}{|c|c|c|}
\hline Constructs & Upstream Primer & Downstream Primer \\
\hline SMF3 promoter -CLN3 & $\begin{array}{l}\text { 5'-ACTCTCGTTCAAGACACTGATTTGATACGCTTTC } \\
\text { TGTACGCGTCCATATCCCTCATATACATG-3' }\end{array}$ & $\begin{array}{l}\text { 5'-CATTAGCGTATCTAATTATGGTATCCTTCAATAT } \\
\text { GGCCATITTTCTCTTGTGATGCTACTACTG-3' }\end{array}$ \\
\hline CPA1 promoter -CLN3 & $\begin{array}{l}\text { 5'-ACTCTCGTTCAAGACACTGATTTGATACGCTTTC } \\
\text { TGTACGCGGGAAATTCATTGTGCACAA- } \text { 3' }^{\prime}\end{array}$ & $\begin{array}{l}\text { 5'-CATTAGCGTATCTAATTATGGTATCTTTCAATAT } \\
\text { GGCCATITGAAATATTTTTAGGAGTGGT-3' }\end{array}$ \\
\hline LSR1 promoter -RPB4 & $\begin{array}{l}\text { 5'-TTCATCCGTATTGAAAATATATATATAAGAATATAG } \\
\text { AAAAGTTTCAAGAAAGAAAACCCAC-3' }\end{array}$ & $\begin{array}{l}\text { 5'-TTCTCCGTCTTGTTTGAAAGGTTGATGTAGAA } \\
\text { ACATTCATATTCGTGGGGATTTAAAAAA-3' }\end{array}$ \\
\hline
\end{tabular}

metabolism. Prog. Nucleic Acid Res. Mol. Biol. 73:221-250.

7. Gasch, A.P., P.T. Spellman, C.M. Kao, O. Carmel-Harel, M.B. Eisen, G. Storz, D. Botstein, and P.O. Brown. 2000. Genomic expression programs in the response of yeast cells to environmental changes. Mol. Biol. Cell 11:4241-4257.

8. Parviz, F. and W. Heideman. 1998. Growthindependent regulation of $C L N 3$ mRNA levels by nutrients in Saccharomyces cerevisiae. J. Bacteriol. 180:225-230.

9. Ares, M., Jr. 1986. U2 RNA from yeast is unexpectedly large and contains homology

\section{A}

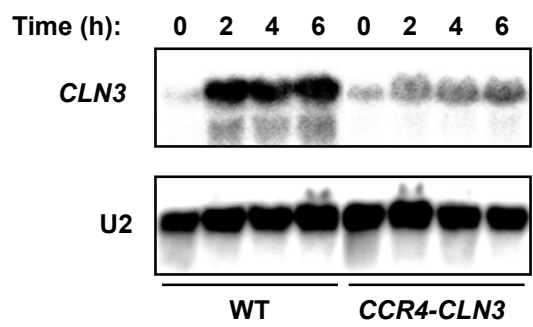

B

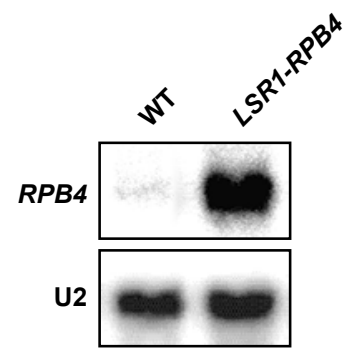

C

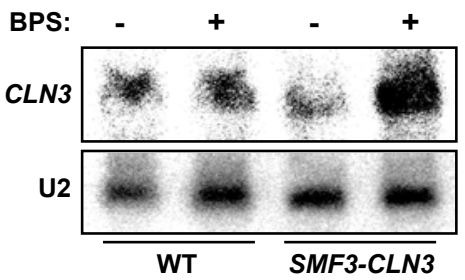

to vertebrate $\mathrm{U} 4, \mathrm{U} 5$, and $\mathrm{U} 6$ small nuclear RNAs. Cell 47:49-59.

10. Jensen, G.J., G. Meredith, D.A. Bushnell, and R.D. Kornberg. 1998. Structure of wildtype yeast RNA polymerase II and location of Rpb4 and Rpb7. EMBO J. 17:2353-2358.

11. Chen, X.Z., J.B. Peng, A. Cohen, H. Nelson, N. Nelson, and M.A. Hediger. 1999. Yeast SMF1 mediates $\mathrm{H}(+)$-coupled iron uptake with concomitant uncoupled cation currents. J. Biol. Chem. 274:35089-35094.

12.Portnoy, M.E., L.T. Jensen, and V.C. Culotta. 2002. The distinct methods by which

Figure 2. Examples demonstrating altered gene expression after heterologous promoter insertion. (A) Northern blot detection of $C L N 3$ expression levels in wild-type and CCR4-CLN3 strains. Wild-type (BY4742) and the isogenic CCR4-CLN3 promoter fusion (DP2) strains were grown for $48 \mathrm{~h}$ to post-log phase $\left(6 \mathrm{~A}_{660}\right)$ in yeast extract peptone dextrose (YEPD) medium and transferred to fresh YEPD at an $\mathrm{A}_{660}$ of 0.4. Samples were collected at the indicated time points for RNA preparation and Northern blot analysis. For Northern blot analysis, RNA was isolated (16) and probed with a 1.8-kb BamHI fragment from $C L N 3$. Blots were probed with a radiolabeled 0.6-kb $\mathrm{SacI}$ fragment from $\mathrm{U} 2$ to confirm uniform loading. (B) Northern blot detection of RPB4 expression levels in wild-type and LSR1-RPB4 strains. Wild-type (BY4742) and the isogenic $L S R 1-R P B 4$ promoter fusion (DLY2) strains were grown to mid-log phase $\left(1 \mathrm{~A}_{660}\right)$ in YEPD medium. Samples were collected for RNA preparation and Northern blot analysis with a 558-bp fragment from $R P B 4$ $\left({ }^{+} 68-{ }^{+} 626\right)$. (C) Northern blot detection of induction of $S M F 3-C L N 3$ by iron chelation. Wildtype (BY4742) and the isogenic SMF3-CLN3 promoter fusion (DLY4) strains were grown to mid-log phase $\left(1 \mathrm{~A}_{660}\right)$ in Synthetic dropout (SD) medium with or without the $1.6 \mu \mathrm{M}$ iron chelator bathophenanthroline-disulfonic acid (BPS) as indicated. Samples were collected for RNA preparation and Northern blot analysis with a $C L N 3$ probe. A U2 probe was used as a loading and transfer control. manganese and iron regulate the Nramp transporters in yeast. Biochem. J. 362:119-124.

13. Delbecq, P., M. Werner, A. Feller, R.K. Filipkowski, F. Messenguy, and A. Pierard. 1994. A segment of mRNA encoding the leader peptide of the CPA1 gene confers repression by arginine on a heterologous yeast gene transcript. Mol. Cell. Biol. 14:2378-2390.

14. Wang, Z., A. Gaba, and M.S. Sachs. 1999. A highly conserved mechanism of regulated ribosome stalling mediated by fungal arginine attenuator peptides that appears independent of the charging status of arginyl-tRNAs. J. Biol. Chem. 274:37565-37574.

15. Waghmare, S.K., V. Caputo, S. Radovic, and C.V. Bruschi. 2003. Specific targeted integration of kanamycin resistance-associated nonselectable DNA in the genome of the yeast Saccharomyces cerevisiae. BioTechniques 34:1024-1033.

16. Hall, D.D., D.D. Markwardt, F. Parviz, and W. Heideman. 1998. Regulation of the Cln3/ Cdc28 Kinase by cAMP in Saccharomyces cerevisiae. EMBO J. 17:4370-4378.

Received 24 February 2006; accepted 4 April 2006.

Address correspondence to Warren Heideman, School of Pharmacy, University of Wisconsin, 425 N. Charter Street, Madison, WI 53706, USA. e-mail: wheidema@facstaff.wisc.edu

To purchase reprints of this article, contact Reprints@BioTechniques.com 